\title{
A APRENDIZAGEM ORGANIZACIONAL: A MUDANÇA COMO CRESCIMENTO NAS ORGANIZAÇÕES PÚBLICAS ${ }^{1}$
}

\author{
Agna Suely Gomes de Oliveira \\ agnasuely@hotmail.com \\ Mestranda em Administração e Gestão Pública - Universidade de Aveiro \\ Leila Maria Chagas Serra \\ leilamaria3@hotmail.com \\ Mestranda em Administração e Gestão Pública - Universidade de Aveiro \\ Doutoranda em Gestão - Ciência Aplicada à Decisão - Universidade de Coimbra
}

\begin{abstract}
Resumo
O presente artigo pretende contribuir para a pertinência do estudo sobre a aprendizagem organizacional. Tal abordagem visa remeter para futuras pesquisas neste âmbito, tendo em vista a sua relevância num mundo contemporâneo considerado competitivo e dinâmico. Está baseado em fundamentações teóricas, tendo como objetivo apresentar conceitos sobre o processo de aprendizagem organizacional, ressaltando a sua importância no contexto das organizações públicas. Para isso, enfatizou-se a importância da prática da aprendizagem e do conhecimento nas organizações públicas, demonstrando como estas organizações podem aprender e transformar os seus espaços num ambiente de aprendizagem, para investir no desenvolvimento das competências e habilidades do seu capital intelectual. Destaca-se, deste modo, a importância dos gestores públicos no processo de aprendizagem da organização, visto que sua confiança e aderência às mudanças propicia um clima organizacional equilibrado e de confiança para os funcionários, motivando-os a aplicar os novos conceitos e promover uma efetiva mudança organizacional. Para clarificar o tema, apresenta-se uma experiência de aprendizagem organizacional numa empresa pública brasileira, demonstrando de que maneira se deu a "internalização" do conhecimento adquirido e aplicação prática dos mesmos no dia a dia da organização. A conclusão evidenciou que a mudança e a flexibilidade nas organizações públicas são objetivos centrais para a aplicação da aprendizagem organizacional, bem como ficou evidenciado que existe a possibilidade de aprendizagem concreta das organizações públicas. Para tanto nesse artigo são destacados, ainda, reflexões preliminares sobre esta problemática.
\end{abstract}

Palavras chave: Aprendizagem organizacional, Organizações públicas, Mudança, Flexibilidade, Gestores públicos

\begin{abstract}
This article aims to contribute to pertinence study the of organizational learning. Such an approach is a link for future research in this area, with a view to its relevance in the contemporary world be competitive and dynamic. It is based on theoretical foundations, aiming to present concepts about the process of organizational learning, highlighting its importance in the context of public organizations. For this, emphasized the importance of the practice of learning and knowledge at the public organizations, demonstrating how these organizations can learn and transform their spaces into a learning environment, to invest in the development of skills and abilities of its intellectual capital. It is noteworthy, therefore, the importance of public managers in the learning process of the organization, as their trust and adherence to organizational climate change provides a balanced and confidence to employees, motivating them to apply new concepts and promote a real change organizational. To clarify the issue, presents an organizational learning experience in a Brazilian public company, showing how it gave the internalization of the acquired knowledge and practical application of these day-to-day organization. The conclusion showed that the change in public organizations and flexibility are key objectives for the implementation of organizational learning, and it was evident that there is a concrete possibility of learning of public organizations. For this are highlighted in this article also preliminary reflections on this issue.
\end{abstract}

Keywords: Organizational learning, Public organizations, Change, Flexibility, Public managers

\footnotetext{
${ }^{1}$ Este artigo resulta de reflexões premilinares de um trabalho (de nossa autoria) apresentado no âmbito na disciplina "Políticas Públicas de Recursos Humanos" ministrada no Mestrado em Administração e Gestão Pública da Universidade de Aveiro.
} 


\section{Introdução}

A maioria das teorias sobre a aprendizagem organizacional foram desenvolvidas com objetivo de fundamentar a aprendizagem organizacional nas organizações privadas, tendo como destaque componentes relacionados à inovação, competitividade e geração de lucros.

Entretanto, tais teorias comprovam, através de muitos autores citados neste artigo que as organizações públicas, mesmo não possuindo objetivos meramente económicos - geração de lucro - mas sim a prestação de serviços públicos à população, podem aprender e transformar os seus espaços num ambiente de aprendizagem, para investir no desenvolvimento das competências e habilidades do seu capital intelectual. Dessa forma, face às novas alterações sociais e económicas, faz-se necessário a adaptação das organizações públicas às novas formas de gestão.

O conceito de aprendizagem organizacional foi incorporado no cenário das teorias de administração em meados da década de 1980, identificando aquelas organizações capazes de adaptar-se aos novos conceitos exigidos pelo mercado. Assim, "uma organização que aprende é uma organização habilitada na criação, na aquisição e na transferência de conhecimento e em modificar o seu comportamento para refletir novos conhecimentos e perceções" (Kiernan, 1998, p. 198).

Diante deste contexto, pode-se indagar quais os reais benefícios e as limitações proporcionados pela utilização da aprendizagem organizacional na administração pública, bem como de que maneira a mudança pode otimizar o processo de aprendizagem organizacional e contribuir para o crescimento das organizações públicas.

Desta forma, o presente artigo tem como objetivo principal contribuir para o esclarecimento do processo da aprendizagem nas organizações públicas, ressaltando os conceitos teóricos e práticos, sua relação com a inovação, mudança, flexibilidade, competência e sua aplicação, bem como conhecer, por meio de uma pesquisa bibliográfica, opiniões de especialistas acerca da importância e do desenvolvimento do profissional na administração pública. Ainda, objetiva apresentar uma experiência de aprendizagem organizacional no âmbito de uma empresa pública brasileira, podendo-se concluir sobre a possibilidade de aprendizagem nas organizações públicas. 


\section{Conceito e importância do processo de Aprendizagem Organizacional}

As mais diversas teorias sobre o conceito de aprendizagem organizacional têm procurado explicar como ocorre o processo de aprendizagem dentro de uma organização, especialmente no tocante a geração, transferência e aplicação do conhecimento. Nesse sentido, é fundamental a existência de condições propícias à aprendizagem: "O processo de aprendizagem, numa organização, não só envolve a elaboração de novos mapas cognitivos, que possibilitem compreender melhor o que está ocorrendo no seu ambiente externo e interno, como também, a definição de novos comportamentos, que comprovam a efetividade da aprendizagem" (Fleury \& Fleury, 1997, p. 20).

Dixon (1994), por sua vez, conceitua que o processo de aprendizagem da organização, o qual deve ser contínuo, passa pelos níveis do indivíduo, do grupo e das organizações, com objetivo de transformar a organização através da satisfação de seus stakeholders (grupos de interesse). Huber (1991) acredita que a aprendizagem de uma organização ocorre se os seus comportamentos são alterados, após o processamento de informações.

No que concerne à aprendizagem individual, a internalização do conhecimento varia entre cada indivíduo, considerando que cada um possui uma diferente perceção da realidade. A aprendizagem individual alia o novo conhecimento a outras estruturas de significação que já existem neste indivíduo. Nesse sentido, pode-se afirmar que o conhecimento é absorvido de acordo com a capacidade individual de aprendizagem (Dixon, 1994).

A organização, por sua vez, pode ser caracterizada como um conjunto de indivíduos, cada um com suas estruturas de significação (Dixon, 1994), interagindo entre si e com o ambiente. Cada indivíduo, por si só, possui capacidade de aprendizagem, mas é a partir da interação coletiva e recíproca que se dá a aprendizagem organizacional.

Então, a aprendizagem da organização dá-se através da existência de várias componentes, tais como um pensamento sistémico, denominado como a quinta disciplina (Senge, 2002), permitindo que a organização, a longo prazo, consolide suas visões e concretize os objetivos planeados inicialmente. Ademais, para a concretização deste pensamento, faz-se necessária a existência de outros componentes, tais como: visão compartida, modelos mentais, aprendizagem em equipa e domínio pessoal para realizar o potencial (Senge, 2002). 
A forma de aprendizagem também está relacionada à capacidade da organização de internalizar os conhecimentos individuais e construir rotinas conforme sua própria cultura (Dodgson, 1993). Em outras palavras, apesar do conhecimento organizacional construir-se a partir da aprendizagem individual, a organização possui um conhecimento próprio e memórias que lhe permite que mantenha certos comportamentos, valores e normas ao longo do tempo, ainda que seus membros tenham sido substituídos (Hedberg, 1981).

Guns (1998, p. 33) acrescenta que "a aquisição de conhecimentos, habilidades, valores, convicções e atitudes que acentuem a manutenção, o crescimento e o desenvolvimento da organização" permitem a aprendizagem coletiva dentro da organização.

Castañeda e Ríos (2007), ainda, entendem serem necessárias três condições para a efetividade do processo de aprendizagem na organização: uma cultura que facilite a aprendizagem, um processo técnico e institucional de formação e capacitação e, por fim, a transferência de informação convertida em conhecimento. Não obstante, o processo de aprendizagem de uma organização pode ocorrer a partir de suas próprias experiências ou da observação de outras organizações (Huber, 1991).

Apesar de ter sido enfatizado que a aprendizagem leva à ação e à incorporação de novas práticas comportamentais à cultura organizacional, há autores que entendem que é possível que a aprendizagem não determine qualquer modificação nos indivíduos ou na própria organização, fazendo com que as organizações aprendam incorretamente ou aprendam corretamente aquilo que é incorreto (Huber, 1991). Um dos motivos para a não incorporação dos conhecimentos às práticas organizacionais deve-se ao facto de que cada indivíduo reage diferentemente aos estímulos externos, levando-os ou não à incorporação de determinadas características ou comportamentos (Dixon, 1994). Convém ressaltar que a aprendizagem organizacional deve ser concebida como um processo em continuidade (Dixon, 1994), que pode, muitas vezes ser imprevisível, dados os obstáculos culturais, económicos e problemas estruturais encontrados ao longo do percurso.

Segundo Kolb (1984), “a aprendizagem organizacional é um processo para toda a vida, determinado pela experiência concreta, pela capacidade de reflexão, conceptualização abstrata e experimentação ativa. Trata-se de um processo cíclico, pelo que planos, ações e conceitos são modificados pela reflexão feita sobre as experiências e não só sobre os resultados". 
Com base no acima exposto, pode-se afirmar que a aprendizagem organizacional é um processo pelo qual uma organização, através de seus membros, adquire conhecimentos, transformando-os em processos e ações que podem, ou não, trazer melhorias aos métodos de produção ou prestação de serviços. Em outras palavras, o aumento do conhecimento da organização significa a aquisição de conhecimentos pelos indivíduos na organização e a incorporação destes nas práticas e rotinas do dia a dia, com objetivo de promover melhoria contínua dos processos.

\section{A mudança: consequência da Aprendizagem Organizacional}

A mudança de comportamento, rotinas ou processos é inerente ao processo de aprendizagem ocorrida na organização. É importante salientar que a absorção do conhecimento está relacionada à sua cultura, tendo em vista que ele será construído e aplicado de acordo com as crenças e valores, gerando rotinas ao redor das atividades desenvolvidas pela organização (Dodgson, 1993).

O processo de mudança e aprendizagem de uma organização deve ser contínuo (Dixon, 1994; Dodgson, 1993), sendo passível de ocorrer em organizações que facilitam, propiciam e encorajam a aprendizagem de seus membros, estendendo essa cultura de aprendizagem aos seus stakeholders (grupos de interesse), valorizando as estratégias políticas de recursos humanos (Dodgson, 1993).

No âmbito da psicologia, a aprendizagem organizacional é vista como uma forma de adaptação aos diversos contextos ambientais da organização, enquanto, no âmbito da gestão, a aprendizagem é um meio necessário para a sua sobrevivência, tendo em vista a possibilidade de propiciar o aumento da competitividade, da produtividade e inovação (Dodgson 1993). Por este motivo, a inovação é um dos principais motivos da aprendizagem organizacional, uma vez que num mercado de consumo, as organizações, sejam públicas ou privadas, tendem a manter-se competitivas a partir de práticas inovadoras e modernas, adaptadas à sociedade em que estão inseridas.

No entanto, é importante ressaltar que não existe um modelo de aprendizagem organizacional ideal e aplicável a todas as organizações. Entretanto, é indispensável considerar as particularidades da organização, analisar a sua cultura, os seus valores e o seu ambiente interno e 
externo para identificar quais são as mudanças necessárias para garantir a aprendizagem organizacional contínua.

Dodgson (1993) relaciona três fatores importantes do processo de aprendizagem organizacional: o conhecimento, as competências específicas e as rotinas. O conhecimento pode ser entendido pelo modo como a organização adquire informações e as articula internamente de acordo com as suas competências específicas, ou seja, a organização internaliza o conhecimento de acordo com os seus valores e crenças, dissipando-o através de novas competências e habilidades. A rotina é, por sua vez, o elemento que permite que a organização crie memórias e bases de conhecimentos, através da geração de normas, procedimentos, tecnologias e culturas. Ela permite que a organização consolide a sua aprendizagem independentemente da aprendizagem individual, o qual permanece mesmo na hipótese da troca ou rotatividade de seus membros - turnover (Dodgson, 1993). Entretanto, a rotina não deve ser um fator limitativo à inovação, sendo certo que a organização deve estar em contínuo processo de aprendizagem e desenvolvimento (Madureira \& Rodrigues, 2006).

Neste processo também é fundamental que cada membro se sinta parte do processo produtivo da organização, reconhecendo a importância de sua função para o adequado funcionamento do todo porque aqueles que se centram unicamente em seu cargo não se sentem responsáveis pelos resultados coletivos, culpando elementos externos aos problemas de desempenho e aos insucessos da organização (Senge, 2002). No processo de aprendizagem de uma organização, deve ser levado em conta que as decisões mais importantes trazem consequências em todas as suas áreas, sendo muitas delas percebidas a longo prazo. Inclusive no campo político, os líderes não conseguem prever as consequências de suas próprias políticas. Contudo, nem sempre essas decisões têm êxito, podendo, até, custar-lhes o cargo. (Senge, 2002).

O erro pode ser visto, então, como a consequência da internalização inadequada do conhecimento, levando a repetição de erros e perda dos padrões de competitividade (Dixon, 1994) ou, numa ótica mais otimista, como condição essencial à aprendizagem pois permite que a organização reflita sobre sua atuação e internalize este erro como parte da melhoria dos seus processos ou rotinas (Dodgson, 1993). Por outro lado, a absorção de novos conhecimentos, através de novas práticas ou do próprio erro leva à organização a um processo de “desaprendizagem” - unlearning - (Hedberg, 1981), ou seja, os novos conhecimentos adquiridos 
fazem com que outros fiquem obsoletos e tenham que ser descartados. Este processo é umas das partes mais importantes na compreensão da aquisição dos novos conhecimentos.

\section{A Aprendizagem nas Organizações Públicas}

Observa-se a rápida transformação da sociedade atual, devido à implantação da diversidade de novas tecnologias (Dodgson, 1993) principalmente relacionadas aos sistemas de comunicação e produção de novos bens de consumo, trazendo como consequência a necessidade de adaptação das organizações às novas necessidades e perfil de consumidor.

A capacidade de adaptação de uma organização às constantes e rápidas mudanças do mercado pode ser traduzida como um meio para que ela se mantenha competitiva. No âmbito das organizações públicas, mais adaptadas às rotinas burocráticas e estabilidade, o ambiente político e económico complexo propicia o aumento das exigências sobre os resultados efetivos da gestão pública, havendo a necessidade de constante atualização do processo de aprendizagem, de modo a garantir a qualidade dos serviços prestados.

Como parte do processo de evolução pessoal e de sistemas organizacionais, é necessário que as organizações e as pessoas passem por um processo de mudança, o que significa reformular antigas regras, conceitos, padrões de comportamento e valores, sendo adotados novos modelos de informação, os quais devem ser constantemente reforçados de modo a serem incorporados no quotidiano da organização (Madureira \& Rodrigues, 2006).

A partir da década de 1980, as organizações públicas de alguns países passaram a reformular seus valores e competências e a prestar um serviço público de excelência, apoiados nos conceitos de mudança, competência e inovação devido ao novo fenómeno organizacional chamado Nova Gestão Pública (Guimarães \& Souza, 2001b).

As organizações públicas, então, são obrigadas a desempenhar novas funções, adaptando-se às necessidades da sociedade, de modo a prestar um serviço de qualidade ao cidadão. Com isso, é evidente que passa a ser necessário inserir na dinâmica organizacional conceitos de inovação, mudança e criatividade, nascendo uma cultura de aprendizagem. Porém, o processo de aprendizagem se estende no tempo, trazendo benefícios a longo prazo, enquanto os investimentos são imediatos. Isto pode ser uma limitação inicial para a prática da aprendizagem organizacional nas organizações públicas, porém não impede a sua adoção e aplicação. 
No que concerne à aprendizagem em organizações públicas, Antonello \& Godoy (2010) destacam que a "aprendizagem é percebida como um processo político no qual os atores investem muitos esforços para influenciar uns aos outros, interpretar a experiência e tentar construir coalizões internas". Assim, percebe-se que a aprendizagem é um meio para que diferentes interesses políticos sejam colocados em prática por meio da difusão de modelos mentais e, geralmente ocorre quando há mudança de governos, momento em que há tendência para a instalação de novas políticas organizacionais, implicando em novas formas e mudança de identidade da organização pública.

Ao contrário do que acontece com as organizações privadas, a aprendizagem das organizações públicas deve ser vista como um meio de criar novas possibilidades para a ação e diálogos públicos (Ventriss \& Luke, 1988). Na verdade, segundo o entendimento de Ventriss \& Luke (1988), as organizações públicas possibilitam a criação de mecanismos políticos democráticos, procurando adequar políticas públicas à melhoria de vida das pessoas e não só implementar as políticas promulgadas. Entretanto, de acordo com os mesmos autores, na formulação e aplicação de políticas públicas, encontram-se alguns obstáculos à internalização da aprendizagem. Dentre eles, destacam-se: (i) a ação pública se dá de maneira dispersa entre os entes políticos, atingindo públicos diversos; (ii) os atores políticos não agem unilateralmente; (iii) os resultados das políticas públicas demoram a aparecer ou desconsideram custos implícitos (Ventriss \& Luke, 1988).

Por este motivo, Ventriss \& Luke (1988) trazem à tona outro aspeto da aprendizagem organizacional da administração pública, o qual refere à necessidade de serem adotadas medidas capazes de avaliar a eficácia das políticas públicas, essencialmente através da avaliação dos cidadãos envolvidos. Esta ação faz com que sejam avaliados os resultados das políticas promovendo um debate entre os interessados na questão e propiciando a melhoria do serviço.

$\mathrm{Na}$ realidade, a prática da mudança tem sido uma estratégia discutida entre os teóricos organizacionais como um facto que intervém na gestão em geral, tanto nas organizações privadas, como nas organizações públicas. Para Motta (1997) “... a gestão contemporânea tornou-se a prática da mudança, tanto na área empresarial quanto no setor público. Nas empresas, as variações tecnológicas e económicas tornam o processo produtivo vulnerável, e conquista-se o progresso em meio a descontinuidades e inovações. Na administração pública, as pressões 
comunitárias por mais e melhores serviços provocam revisões nas funções do Estado, e questionam-se tanto as formas de ação quanto a própria legitimidade das organizações. Na busca de qualidade e eficiência, as organizações públicas se assemelham às empresas privadas" (Motta, 1997, p. 15).

Madureira \& Rodrigues (2006) sugerem também que, no âmbito da administração pública, a mudança organizacional ocorra em todos os níveis, especialmente na alta gestão. Os gestores tem função de encorajar e coordenar a variedade de interação entre a aprendizagem nos mais variados níveis (Dodgson, 1993).

Em outras palavras, a organização, através de seus gestores, devem estar preparadas para a vigência de um novo modelo comportamental. Somente com esse estado é que será possível exigir do funcionário público uma mudança de comportamento e absorção de novos valores, tendo em vista que a insegurança e desmotivação dos gestores podem-se traduzir em desconfiança e desmotivação dos funcionários, fazendo com que a mudança não surta efeitos práticos (Madureira \& Rodrigues, 2006).

Este novo cenário exige do gestor público novas competências, novas habilidades, novos valores, novas estratégias administrativas, novas perceções, novos conhecimentos e novas atitudes estabelecendo a criação de um ambiente organizacional em que o conhecimento, a inovação e as mudanças possam ser socializados como um grande desafio.

Também é comum encontrar resistência à mudança de comportamento nestas organizações públicas. Madureira \& Rodrigues (2006) citam algumas das razões possíveis para a resistência à mudança, por parte dos funcionários: "a perda de privilégios ou de controlo" (que pode acontecer, por exemplo, devido a uma reorganização da estrutura orgânica e das funções), “a incerteza quanto ao futuro" e "a perda da hegemonia das competências" (decorrente, por exemplo, da introdução de um sistema tecnológico que os funcionários não dominam).

Ademais, Madureira \& Rodrigues (2006) entendem que o processo de mudança tende a aparecer de forma planeada e deve incluir novas competências comportamentais para seus membros, como forma de garantir e reforçar a sua confiança e auto-estima. A burocracia, componente existente em todas organizações, não pode ser um entrave à inserção de novos conhecimentos e técnicas que permitem a melhoria dos sistemas organizacionais. 
É necessário que os gestores públicos adotem medidas de modo a aproximar os cidadãos da administração, bem como possibilite a melhoria dos meios e mecanismos para uma prestação eficiente de serviços públicos (Ventriss \& Luke, 1988).

O papel dos gestores públicos é de fundamental importância, visto que sua confiança e adesão às mudanças geram um clima organizacional seguro e de respeito aos funcionários, motivando-os a aplicar os novos conceitos e a promover uma efetiva mudança organizacional. A aprendizagem individual do gestor, por sua vez, só ocorre se permitir uma reflexão crítica e reformulação das metas da organização e escolhas políticas, permitindo o desenvolvimento de novos processos (Ventriss \& Luke, 1988). Neste contexto, a busca da flexibilidade pode representar um diferencial para a mudança nos padrões de gestão das organizações públicas.

Para concluir, destaca-se a apresentação de Queiroz \& Nogueira (2005) sobre o processo de aprendizagem organizacional ocorrido no Instituto Brasileiro de Geografia e Estatística - IBGE. Por ser uma empresa pública responsável pela realização de todas as estatísticas oficiais do governo brasileiro - dados socioeconómicos, níveis salariais e emprego, estrutura produtiva, etc. - não possui concorrentes no mercado. Contudo, este instituto disputa orçamento com outros órgãos do governo, devendo desempenhar com excelência as atividades que lhe foram atribuídas. Numa breve síntese, destacou-se algumas medidas apontadas por Queiroz \& Nogueira (2005), as quais caracterizam o processo de aprendizagem organizacional deste instituto a partir dos cinco caminhos propostos por Fleury \& Fleury (1997) onde pode ocorrer a aprendizagem organizacional.

Como forma de resolver problemas sistemáticos e encontrar soluções para as deficiências da organização, o IBGE promoveu uma pesquisa com seus funcionários, cujo diagnóstico proporcionou a melhoria na estrutura organizacional e otimização das relações entre as suas diversas unidades (resolução sistemática de problemas). Não obstante, o IBGE, com objetivo de implantar novos conhecimentos na organização através do método científico, apostou no desenvolvimento de um sistema de Gestão de Suprimentos de Fundos, cujo objetivo foi solucionar entraves nas áreas administrativas e financeiras, o qual obteve grande êxito (experimentação). 
Da mesma maneira, uma medida inicialmente experimental foi adotada de modo permanente, a qual consistiu em promover um processo seletivo interno com finalidade de destacar novos talentos e selecionar novos chefes das unidades regionais (experiências passadas). Tal medida foi fundamental para a revelação de novos talentos e promoção de ascensão profissional baseada no mérito, visto que os cargos anteriores eram preenchidos através de indicação da diretoria nacional. Ficou evidente, com isso, que o IBGE promoveu uma aprendizagem com base na reavaliação dos sucessos e fracassos das experiências passadas, logrando êxito.

Não obstante, com a aquisição de um novo software (Lotus Notes), o instituto promoveu grande circulação de ideias e conhecimento, gerando a circulação do conhecimento, proporcionando a aprendizagem coletiva (circulação de conhecimento). Outra ferramenta de aprendizagem foi a observação de experiências realizadas por outras instituições e que, posteriormente foi internalizada pelo IBGE - a contratação de um curso (Curso de Desenvolvimento de Habilidades de Pesquisa) com objetivo de aumentar o conhecimento individual quanto às práticas estatísticas, o qual havia sido utilizado com sucesso por um instituto canadense de estatística (experiências realizadas por outros).

Deste modo, ficou demonstrado que a aprendizagem organizacional promovida dentro do instituto propiciou melhoria no processo de trabalho dos funcionários do IBGE, garantindo o aprimoramento do desempenho do órgão face à evolução da sociedade.

\section{Conclusão}

Após uma breve apresentação de conceitos teóricos relacionados à aprendizagem individual e organizacional, pôde-se descrever o processo de mudança como parte da aprendizagem de uma organização, seja ela pública ou privada.

Apesar de uma rápida análise sobre um tema tão complexo e pertinente, pode-se concluir que o processo de aprendizagem organizacional requer envolvimento individual dos membros da organização, os quais, de maneira coletiva, disseminam o conhecimento. Uma vez consolidado o conhecimento, diz-se que houve sedimentação de uma cultura organizacional, através da criação de novas práticas e rotinas de trabalho.

Assim, percebe-se que a aprendizagem organizacional tornou-se um fenómeno que altera a situação da coletividade mediante o contexto em que está inserida, sendo um processo 
permanentemente contínuo que, de facto, apresenta mecanismos sobre os quais se alicerça a transformação do conhecimento e da aprendizagem.

Por outro lado, ficou evidente que o processo de aprendizagem das organizações públicas é muito diferente das privadas, o que é natural, tendo em vista que seu objetivo maior não é a geração de lucro ou manutenção da competitividade, mas sim a realização e criação de processos que permitam o desenvolvimento socioeconómico do Estado, onde a burocracia é mais predominante. Contudo, foram apresentadas ideias sobre o processo de internalização do conhecimento nas organizações públicas, demonstrando-se, inclusive, uma experiência de sucesso no Instituto Brasileiro de Geografia e Estatística, conforme trabalho científico de Queiroz \& Nogueira (2005).

Entretanto, vale ressaltar que as organizações públicas estão mais atentas às mudanças da sociedade, visto que estão a inovar e adquirir constantemente novos conhecimentos organizacionais com o objetivo de melhorar continuamente a prestação dos serviços.

Com base nas experiências trazidas por Queiroz \& Nogueira (2005), pode-se dizer que houve aprendizagem organizacional no Instituto Brasileiro de Geografia e Estatística, uma vez que foram consolidados novos processos, práticas e rotinas a partir da observação da realidade da organização. Ademais, investiu-se em formação e capacitação dos funcionários, bem como foi desenvolvida nova ferramenta institucional, a qual possibilitou a disseminação do conhecimento.

É evidente que a aprendizagem, a qualquer nível - individual ou organizacional - a qualquer tempo exige esforços, pois requer que novos valores e conceitos sejam incorporados e antigos comportamentos deixados de lado. Tendo-se em conta, entretanto, o rápido desenvolvimento da sociedade e das novas tecnologias, fica claro que a aprendizagem está a ocorrer mais rapidamente a cada dia.

Desta maneira, fica claro que, com a adoção de novos sistemas, rotinas, estratégias de desenvolvimento e novas ferramentas de gestão, todas as organizações, inclusive as públicas, podem aprender. Não obstante, não existe um modelo de aprendizagem organizacional ideal para todas as organizações, uma vez que cada uma possui suas próprias características. Porém, é imprescindível considerar as particularidades da organização, analisar a sua cultura, a sua gestão, os seus valores e as suas relações com o ambiente interno e externo, para identificar quais as 
Investigação e Intervenção em Recursos Humanos 2011 - Gestão para a cidadania

Escola Superior de Estudos Industriais e de Gestão do Instituto Politécnico do Porto

27 e 28 de Outubro de 2011

mudanças que serão necessárias para garantir a coletividade da construção e da socialização da

aprendizagem, do conhecimento e da inovação. Em detrimento à dinâmica do cenário social, considera-se relevante o aprofundamento desta pesquisa no âmbito académico.

\section{Referências Bibliográficas}

ARGYRIS, Chris; SCHÖN, Donald (1978) - Organizational learning: a theory of action perspective. Reading, Massachusetts: Addison-Wesley.

ANTONELlO, Claudia Simone; GODOY, Arilda Schimidt (2010). A encruzilhada da aprendizagem organizacional: uma visão multiparadigmática. "Revista de Administração Contemporânea", Curitiba, [Em linha]. Vol.14, n. 2 (Mar/Abr 2010), art. 7, p 310-332 [Consult. 01 de Abril 2010]. Disponível na Internet em: http://www.anpad.org.br/periodicos/content/frame_base.php?revista=1. ISSN 1982-7849.

CASTAÑEDA, Délio Ignácio; RIOS, Maniel Fernandez (2007). Validación de una escala de niveles y condiciones de aprendizaje organizacional. "Revista Javeriana", Bogotá [Em linha]. Vol.6, n. ${ }^{\circ} 2$ (Mai/Ago 2007), p 245-254 [Consult. 01 de Abril 2010]. Disponível na Internet: http://revistas.javeriana.edu.co/index.php/revPsycho/article/view/113/98. ISSN 1657-9267.

DIXON, N. M. (1994) - The organizational learning cycle: how we can learn collectively. London: McGrawHill.

DODGSON, Mark. (1993). Organizational learning: A review of some literatures. "Organizational Studies", Brighton [Em linha]. Vol.14, n. 3 (Mai 1993), p 375-394 [Consult. 01 de Abril 2010]. Disponível na Internet: http://oss.sagepub.com/content/14/3/375.abstract. ISSN 0170-8406.

FLEURY, Afonso; FLEURY, Maria T. L. (1997) - Aprendizagem e inovação organizacional: As experiências de Japão, Coréia e Brasil. ( $2^{\mathrm{a}}$ ed.), São Paulo: Atlas.

GUIMARÃES, T.A.; SOUZA, Eda Castro L. de (2001b). Aprendizagem organizacional na nova administração pública. "R\&D Management", Rio de Janeiro [Em linha]. Vol.31, n. 3 (2001b), p 249-255 [Consult. 01 de Abril 2010]. Disponível na Internet: http://pt.scribd.com/doc/6189509/Tomas-de-Aquino-Guimaraes-RD-Management-v31.

GUNS, Bob. (1998) - A organização que aprende rápido: seja competitivo utilizando o aprendizado organizacional. São Paulo: Futura.

HEDBERG, B. (1981). How organizations learn and unlearn, in NYSTRÖM, P.; STARBUCK W. (Orgs.), Handbook of organizational design, Vol.1, n. ${ }^{\circ}$ 3, p 8, ISBN 019-827241-3. New York: Oxford University Press.

HUBER, Georg P. (1991). Organizational Learning: The Contributing Processes and the Literatures. "Organization Science", Austin [Em linha]. Vol.2, n. ${ }^{\circ} 1$ (Fev 1991), p 88-115 [Consult. 01 de Abril 2010]. Disponível na Internet: http://www.jstor.org/pss/2634941. ISSN 1047-7039.

KIERNAN, Matthew J. (1998) - Os 11 mandamentos da administração do século XXI. São Paulo: Makron Books.

KOLB, D. A. (1984) - Experimental learning: experience as the source of learning. New Jersey: Prentice-Hall.

MADUREIRA, César; RODRIGUES, Miguel. (2006). A Administração Pública do século XXI: Aprendizagem organizacional, mudança comportamental e reforma administrativa. "Comportamento Organizacional e Gestão", Lisboa [Em linha]. Vol.12, n. ${ }^{\circ} 2$ (2006), p 153-171 [Consult. 01 de Abril 2010]. Disponível na Internet: http://www.scielo.oces.mctes.pt/scielo.php?pid=S0872-96622006000200002\&script=sci_arttext. ISSN 0872-9662.

MOTTA, P. R. (1997) - Gestão contemporânea: A teoria e a prática de inovar. Rio de Janeiro: Qualitymark.

QUEIROZ, Marcos A. C. de, \& NOGUEIRA, Mirian Garcia (2005). Aprendizagem organizacional em institutos nacionais de estatística: O exemplo brasileiro. XII SIMPEP - Bauru, São Paulo, 7 a 9 de Novembro de 2005. Disponível na Internet: http://www.simpep.feb.unesp.br/anais_simpep_aux.php?e=12. ISSN 1809-7189.

SENGE, P. M. (2002) - A Quinta disciplina: arte e prática da organização que aprende. 10 ed. São Paulo: Nova Cultural.

VENTRISS, Curtis, \& LUKE, Jeff. (1988). Organizational learning and public policy: towards a substantive perspective. "American Review of Public Administration" [Em linha]. Vol.18, n. 4 (Dez 1988), p 337-357 [Consult. 01 de Abril 2010]. Disponível na Internet: http://arp.sagepub.com/content/18/4/337. ISSN 0275-0740. 